\title{
9 \\ Serosorting and recreational drug use are risk factors for diagnosis of genital infection with chlamydia and gonorrhoea among HIV-positive men who have sex with men: results from a clinical cohort in Ontario, Canada
}

\author{
Ramandip Grewal, ${ }^{1}$ Vanessa G Allen, ${ }^{2}$ Sandra Gardner, ${ }^{3,4}$ Veronika Moravan, ${ }^{5}$ \\ Darrell H S Tan, 6,7,8 Janet Raboud, ${ }^{3,7}$ Ahmed M Bayoumi, ${ }^{1,6,7,8}$ Rupert Kaul, ${ }^{6}$ \\ Tony Mazzulli, ${ }^{2}{ }^{11}$ Frank McGee, ${ }^{12}$ Sean B Rourke, ${ }^{1,5,13}$ Ann N Burchell, 1,3,14 \\ in collaboration with the OHTN Cohort Study Research Team
}

For numbered affiliations see end of article.

\section{Correspondence to}

Dr Ann N Burchell, Department of Family and Community Medicine and Li Ka Shing Knowledge Institute, St. Michael's Hospital, 30 Bond Street, Toronto, Ontario, Canada M5B 1W8; burchella@smh.ca

Received 28 December 2015 Revised 31 March 2016 Accepted 16 April 2016
CrossMark

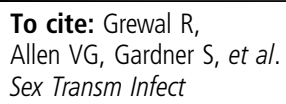

\section{ABSTRACT}

Objectives Rates of chlamydia and gonorrhoea have been rising in urban centres in Canada, particularly among HIV-positive men who have sex with men (MSM). Our objective was to identify behavioural risk factors for diagnosis with chlamydia and gonorrhoea in this population, with a focus on the HIV status of sexual partners.

Methods The OHTN Cohort Study follows people in HIV care across Ontario. We restricted the analysis to 1997 MSM who completed questionnaires in 20102013 at one of seven clinics that submit all chlamydia and gonorrhoea tests to the provincial public health laboratory; we obtained test results via record linkage. We estimated cumulative incidences using Kaplan-Meier methods and identified risk factors for diagnosis of a composite outcome (chlamydia or gonorrhoea infection) using Cox regression.

Results At follow-up, there were 74 new chlamydia/ gonorrhoea diagnoses with a 12-month cumulative incidence of $1.7 \%(95 \% \mathrm{Cl} 1.1 \%$ to $2.2 \%)$. Risk factors for chlamydia/gonorrhoea diagnosis were: $5+$ HIV-positive partners ( $\mathrm{HR}=3.3,95 \% \mathrm{Cl} 1.4$ to 7.8 ; reference=none) and recreational drug use $(H R=2.2$, $95 \% \mathrm{Cl} 1.2$ to 3.9$)$.

Conclusions Heightened risks with recreational drug use and multiple HIV-positive partners suggest that chlamydia/gonorrhoea may have achieved high prevalence in certain sexual networks among HIVpositive MSM. Interventions to promote safer sex and timely testing among MSM are needed.

\section{INTRODUCTION}

Rates of chlamydia and gonorrhoea have risen in urban centres in Canada including an increase in case reports among men who have sex with men (MSM). ${ }^{1}$ Coinfection with HIV is particularly concerning because sexually transmitted infections (STIs) can increase likelihood of HIV transmission. ${ }^{1}$ In Ontario, Canada, we previously reported demographic and clinical risk factors for new chlamydia and gonorrhoea diagnoses among an HIV clinical cohort followed from 2008 to $2011 .^{2}$ Risk was highest among MSM, particularly among younger MSM and (for gonorrhoea) among MSM with unsuppressed viral load, with little variation between ethnic groups. ${ }^{2}$ However, full characterisation of risk requires examination of behavioural risk factors since sexual behaviour and prevalence in sexual networks drive STI risk. 'Serosorting', selecting sexual partners of the same HIV serostatus, could raise STI risk since condoms may be used less frequently, which could concentrate infection in sexual networks of HIV-positive persons. ${ }^{3}$ Recreational drug use has also been linked to sexual risk taking. ${ }^{145}$ Our objective was to extend our previous analysis by longitudinally exploring behavioural risk factors for diagnoses of genital chlamydia and gonorrhoea infection among HIV-positive MSM attending HIV care in Ontario, specifically focusing on the risks associated with serosorting and recreational drug use.

\section{METHODS}

We analysed data from the Ontario HIV Treatment Network Cohort Study (OCS) which follows persons attending $10 \mathrm{HIV}$ clinics across Ontario. The province has publicly funded, universal access to medically necessary healthcare. The OCS has been described in detail elsewhere ${ }^{6}$ and received ethical approval from the University of Toronto HIV Research Ethics Board (protocol reference 23954) and participating sites. Participation is voluntary. Data were collected through chart review, annual interviews and record linkage with the Public Health Ontario Laboratories (PHOL). At PHOL, simultaneous cotesting of chlamydia and gonorrhoea urine specimens was done by nucleic acid amplification testing using the Gen-Probe Aptima assay (Gen-Prose, San Diego, California, USA). Canadian guidelines recommend chlamydia and gonorrhoea testing annually for MSM at ongoing risk, regardless of HIV status. ${ }^{1}$

Our focus was on sexual behaviour and recreational drug use risk factors, adjusting for age, region and time-updated viral load as potential confounders. Men reported recreational drug use 
Table 1 Characteristics of HIV-positive men who have sex with men (MSM) at completion of their first sexual behaviour questionnaire, OHTN Cohort Study, 2010-2013

\begin{tabular}{|c|c|}
\hline Total N & $1997(100 \%)$ \\
\hline Mean age at baseline (SD) & $47.7(10.2)$ \\
\hline \multicolumn{2}{|l|}{ Self-reported sexual orientation } \\
\hline Gay & $1767(88.5 \%)$ \\
\hline Bisexual & $151(7.6 \%)$ \\
\hline Heterosexual & $58(2.9 \%)$ \\
\hline Unknown* & $21(1.1 \%)$ \\
\hline \multicolumn{2}{|l|}{ Race/ethnicity } \\
\hline White & $1492(74.7 \%)$ \\
\hline Black/African & $80(4.0 \%)$ \\
\hline Mixed race/ethnicity & $168(8.4 \%)$ \\
\hline Indigenous & $85(4.3 \%)$ \\
\hline Other & $170(8.5 \%)$ \\
\hline \multicolumn{2}{|l|}{ Education } \\
\hline High school or less & $448(22.4 \%)$ \\
\hline Some postsecondary & $435(21.8 \%)$ \\
\hline Completed postsecondary & $1111(55.6 \%)$ \\
\hline \multicolumn{2}{|l|}{ Annual personal income } \\
\hline$<\$ C 20000$ & $702(35.2 \%)$ \\
\hline \$C20 000-\$C59 999 & $794(39.8 \%)$ \\
\hline$\geq \$ C 60000$ & $470(23.5 \%)$ \\
\hline Unknown & $31(1.6 \%)$ \\
\hline \multicolumn{2}{|l|}{ Region where receiving HIV care } \\
\hline Toronto & $1703(85.3 \%)$ \\
\hline Other & $294(14.7 \%)$ \\
\hline \multicolumn{2}{|l|}{ Year of HIV diagnosis } \\
\hline Prior to 2000 & $1129(56.5 \%)$ \\
\hline $2000-2009$ & $737(36.9 \%)$ \\
\hline 2010 or later & $131(6.6 \%)$ \\
\hline Median (IQR) & 1997 (1991-2005) \\
\hline \multicolumn{2}{|l|}{ HIV clinical status } \\
\hline Initiated antiretroviral treatment & $1647(82.5 \%)$ \\
\hline Mean CD4 cell count $/ \mathrm{mm}^{3}$ (SD) & $537(253)$ \\
\hline Undetectable viral load ( $<40$ copies $/ \mathrm{mL}$ ) & $1568(78.5 \%)$ \\
\hline \multicolumn{2}{|c|}{ Recreational drug use in the preceding 6 months } \\
\hline Anyt & $418(20.9 \%)$ \\
\hline Methamphetamines & $188(9.4 \%)$ \\
\hline Cocaine & $205(10.3 \%)$ \\
\hline Club drugs $\ddagger$ & $217(10.9 \%)$ \\
\hline Other§ & $187(9.4 \%)$ \\
\hline Multiple (two or more) & $232(11.6 \%)$ \\
\hline \multicolumn{2}{|l|}{ Sexual behaviours in the preceding 3 months } \\
\hline \multicolumn{2}{|l|}{ Number of sexual partners } \\
\hline None & $710(35.6 \%)$ \\
\hline One & $535(26.8 \%)$ \\
\hline Two to four & $452(22.6 \%)$ \\
\hline Five or more & $274(13.7 \%)$ \\
\hline \multicolumn{2}{|l|}{ Number of HIV-positive partners } \\
\hline None & $1210(60.6 \%)$ \\
\hline One & $330(16.5 \%)$ \\
\hline Two to four & $255(12.8 \%)$ \\
\hline Five or more & $100(5.0 \%)$ \\
\hline \multicolumn{2}{|c|}{ Number of HIV-negative/status unknown partners } \\
\hline None & $1101(55.1 \%)$ \\
\hline One & $364(18.2 \%)$ \\
\hline Two to four & $229(11.5 \%)$ \\
\hline Five or more & $214(10.7 \%)$ \\
\hline
\end{tabular}

Table 1 Continued

\begin{tabular}{|c|c|}
\hline \multicolumn{2}{|l|}{ Anal sex } \\
\hline No partner & $710(35.6 \%)$ \\
\hline Sexually active but no anal sex & $319(16.0 \%)$ \\
\hline Anal sex always with a condom & $349(17.5 \%)$ \\
\hline Any condomless anal sex & $551(27.6 \%)$ \\
\hline \multicolumn{2}{|l|}{ Anal sex with HIV-positive partners 9} \\
\hline No HIV-positive partners & $1210(60.6 \%)$ \\
\hline Sexually active but no anal sex & $124(6.2 \%)$ \\
\hline Anal sex always with a condom & $147(7.4 \%)$ \\
\hline Any condomless anal sex & $416(20.8 \%)$ \\
\hline \multicolumn{2}{|c|}{ Anal sex with HIV-negative/status unknown partners } \\
\hline No HIV-negative/status unknown partners & $1101(55.1 \%)$ \\
\hline Sexually active but no anal sex & $214(10.7 \%)$ \\
\hline Anal sex always with a condom & $347(17.4 \%)$ \\
\hline Any condomless anal sex & $257(12.9 \%)$ \\
\hline \multicolumn{2}{|c|}{$\begin{array}{l}\text { *Only } 0.1 \% \text { of the sample had missing data for race/ethnicity and } 0.2 \% \text { for education. } \\
\text { †Any recreational drug use includes anabolic steroids, amphetamines, } \\
\text { methamphetamines, cocaine, crack/freebase, club drugs, heroin, other opiates, } \\
\text { tranquilisers and other drugs, except cannabis as it was unmeasured for } 4 / 7 \\
\text { participating clinic sites. } \\
\text { ¥Club drugs include Ecstasy/MDMA (3,4-methylenedioxy-methamphetamine), Special } \\
\text { K (ketamine), GHB ( } \gamma \text { hydroxybutyrate), PCP (phencyclidine) and poppers (amyl } \\
\text { nitrite). } \\
\text { §Other includes anabolic steroids, amphetamines, crack/freebase, heroin, other } \\
\text { opiates, tranquilisers and 'other' option in drug use section of questionnaire. } \\
\text { १Serostatus-specific variables were not mutually exclusive. The sexual behaviour } \\
\text { questionnaire had a series of questions for HIV-positive partners followed by } \\
\text { questions specific to HIV-negative/status unknown partners allowing for the capture } \\
\text { and coding of different sexual practices by HIV status of partners. }\end{array}$} \\
\hline
\end{tabular}

in face-to-face interviews. Next, they self-completed a computerised sexual behaviour questionnaire without interviewers present.

As of 12/2013, there were 6408 enrolees. Our analysis included 1997 male participants reporting non-heterosexual orientation or sex with men as an HIV risk factor; selfcompleted at least one sexual behaviour questionnaire between 2010 and 2013 and attended one of seven clinics that submitted all chlamydia and gonorrhoea tests to PHOL, the primary provider for chlamydia and gonorrhoea tests for sexual health clinics (HIV specialty and primary care clinics may also send specimens to private laboratories).

We conducted all statistical analyses using SAS V.9.3 (SAS Institute, Cary, North Carolina, USA). $p$ Values were two-sided and statistical significance was determined using the $\mathrm{p}$ value of $<0.05$. We used Kaplan-Meier methods to estimate the cumulative incidence proportions tested for or diagnosed with chlamydia or gonorrhoea following completion of the first sexual behaviour questionnaire. As previously done, diagnosis rates were calculated among tested and untested participants ${ }^{2}$ thus underestimating true incidence since asymptomatic infections among those untested are excluded from the numerator.

For risk factor analysis, we grouped chlamydia and gonorrhoea into a composite outcome to improve statistical power and because their modes of transmission and clinical/public health implications are similar; HR estimates for separate chlamydia and gonorrhoea outcomes were not meaningfully different. For men diagnosed more than once, we selected the earliest event, which occurred rarely. We excluded participants with missing data (1.3\% of observations) and two cases with zero event times. Men with no events were censored using the earliest of the dates of loss to follow-up, death or 31 December 2013. 


\section{RESULTS}

At completion of their first sexual behaviour questionnaire, men were aged 48 years, on average (table 1 ). The median number of years since HIV diagnosis was 18 (IQR 10-24). The majority $(64.4 \%)$ were sexually active in the preceding 3 months, whereas a minority (20.9\%) reported recreational drug use (table 1).

Men were followed a median of 2.9 years (IQR 1.8-3.3) for a sum of 5833 person-years. The Kaplan-Meier cumulative probabilities of testing for chlamydia/gonorrhoea were $24.6 \%$ at 12 months and $33.1 \%$ at 24 months. In all, 35.1\% (700/1997) were tested over the entire course of follow-up. The majority of tests were ordered by participating HIV clinics $(87.6 \%)$ with only $3.0 \%$ ordered by sexual health/STI/community health clinics and $9.3 \%$ by other health providers. Stratum-specific 12 -month testing probabilities ranged from $15.8 \%$ among sexually inactive men to $42.5 \%$ among men reporting $5+$ partners (table 2).

At follow-up, 41, 46 and 74 men were diagnosed with chlamydia, gonorrhoea and either pathogen, respectively. The Kaplan-Meier 12-month cumulative incidence proportions were $1.0 \%$ (95\% CI $0.6 \%$ to $1.4 \%$ ) for chlamydia, $1.2 \%$ (95\% CI $0.7 \%$ to $1.7 \%$ ) for gonorrhoea and $1.7 \%$ (95\% CI $1.1 \%$ to 2.2\%) for the composite outcome. Among cases, 53\% (39 of 74) had a history of syphilis compared with $9.5 \%$ among non-cases.

Multiple sex partners and recreational drug use were significant risk factors for diagnosis (table 2). Risk was elevated

Table 2 Behavioural risk factors for a diagnosis of genital chlamydia/gonorrhoea infection at follow-up among HIV-positive MSM, OHTN Cohort Study, 2010-2013

\begin{tabular}{|c|c|c|c|c|c|}
\hline Sexual behaviours in preceding 3 months* & $\begin{array}{l}\text { Cumulative } \\
\text { probability } \\
\text { of testing } \\
\text { at } 12 \text { months } \\
(95 \% \mathrm{Cl}) \dagger\end{array}$ & $\begin{array}{l}\text { Cumulative } \\
\text { incidence of } \\
\text { diagnosis } \\
\text { at } 12 \text { months } \\
(95 \% \mathrm{Cl}) \dagger\end{array}$ & $\begin{array}{l}\text { Unadjusted HR } \\
(95 \% \mathrm{Cl})\end{array}$ & $\begin{array}{l}\text { Multivariable } \\
\text { model Ał: } \\
\text { adjusted HR } \\
(95 \% \mathrm{Cl})\end{array}$ & $\begin{array}{l}\text { Multivariable } \\
\text { model B } \neq \text { : } \\
\text { adjusted HR } \\
(95 \% \mathrm{Cl})\end{array}$ \\
\hline \multicolumn{6}{|l|}{ Number of sexual partners } \\
\hline None & $15.8(13.3$ to 18.4$)$ & $0.4(0.0$ to 0.8$)$ & Reference & Reference & \\
\hline One & $20.0(16.7$ to 23.3$)$ & $0.7(0.0$ to 1.4$)$ & $2.7(0.9$ to 7.9$)$ & & \\
\hline Two to four & 33.9 (29.7 to 38.1$)$ & $3.0(1.5$ to 4.6$)$ & 10.3 (4.0 to 26.6$)$ & 3.1 (1.4 to 6.6$)$ & \\
\hline Five or more & 42.5 (36.7 to 48.3$)$ & $4.8(2.4$ to 7.3$)$ & 16.0 (6.1 to 41.7$)$ & 3.7 (1.7 to 8.3$)$ & \\
\hline \multicolumn{6}{|l|}{ Number of HIV-positive partners } \\
\hline None & $19.0(16.9$ to 21.2$)$ & $0.8(0.3$ to 1.2$)$ & Reference & & Reference \\
\hline One & 26.8 (22.2 to 31.4$)$ & $2.2(0.7$ to 3.8$)$ & $2.3(1.1$ to 4.7$)$ & & \\
\hline Two to four & 40.1 (34.2 to 45.9$)$ & $2.9(0.9$ to 5.0$)$ & $5.0(2.7$ to 9.4$)$ & & $1.6(0.7$ to 3.6$)$ \\
\hline Five or more & 42.3 (32.8 to 51.7$)$ & $8.3(3.1$ to 13.5$)$ & 13.5 (7.1 to 25.5$)$ & & $3.3(1.4$ to 7.8$)$ \\
\hline \multicolumn{6}{|l|}{ Number of HIV-negative/status UK partners } \\
\hline None & 20.9 (18.6 to 23.2$)$ & $1.1(0.5$ to 1.7$)$ & Reference & & Reference \\
\hline One & $22.3(18.2$ to 26.4$)$ & $1.0(0.0$ to 2.0$)$ & $1.0(0.5$ to 2.0$)$ & & \\
\hline Two to four & 34.6 (28.6 to 40.5$)$ & $2.5(0.5$ to 4.4$)$ & $2.2(1.2$ to 4.3$)$ & & $1.2(0.5$ to 2.9$)$ \\
\hline Five or more & 33.7 (27.6 to 39.9$)$ & $4.8(2.0$ to 7.5$)$ & 3.5 (1.9 to 6.2$)$ & & $1.2(0.5$ to 3.0$)$ \\
\hline \multicolumn{6}{|l|}{ Anal sex } \\
\hline No partner & $15.8(13.3$ to 18.4$)$ & $1.0(0.3$ to 1.7$)$ & Reference & Reference & \\
\hline Sexually active but no anal sex§ & $20.8(16.5$ to 25.1$)$ & $1.3(0.0$ to 2.5$)$ & $1.9(1.1$ to 3.3$)$ & $0.5(0.1$ to 3.0$)$ & \\
\hline Anal sex always with a condom & $25.6(21.2$ to 30.0$)$ & $3.4(1.5$ to 5.3$)$ & $1.9(0.5$ to 6.9$)$ & $3.3(1.0$ to 11.4$)$ & \\
\hline Any condomless anal sex & $36.8(32.9,40.7)$ & $6.0(4.1$ to 8.0$)$ & $7.0(2.5$ to 19.0$)$ & $3.6(1.0$ to 12.3$)$ & \\
\hline \multicolumn{6}{|l|}{ Anal sex with HIV+ partner } \\
\hline No HIV+ partner & $19.0(16.9$ to 21.2$)$ & $0.8(0.3$ to 1.2$)$ & Reference & & Reference \\
\hline Sexually active with HIV+ partner but no anal sex§ & 17.1 (10.7 to 23.5$)$ & $0.0(0.0$ to 0.0$)$ & $1.0(0.2$ to 4.4$)$ & & $1.2(0.3$ to 5.2$)$ \\
\hline Anal sex with HIV+ partner always with a condom & $28.5(21.5$ to 35.4$)$ & $1.2(0.0$ to 2.8$)$ & 4.0 (1.8 to 8.8$)$ & & $2.8(0.6$ to 13.4$)$ \\
\hline Any condomless anal sex with HIV+ partner & 40.6 (35.9 to 45.2$)$ & $5.4(3.3$ to 7.5$)$ & $6.3(3.7$ to 10.8$)$ & & $2.2(0.5$ to 9.8$)$ \\
\hline \multicolumn{6}{|l|}{ Anal sex with HIV-IUK partner } \\
\hline No HIV-/UK partner & 20.9 (18.6 to 23.2$)$ & $1.1(0.5$ to 1.7$)$ & Reference & & Reference \\
\hline Sexually active with HIV-/UK partner but no anal sex§ & $22.3(16.9$ to 27.7$)$ & $0.4(0.0$ to 1.3$)$ & $0.7(0.2$ to 2.0$)$ & & $1.6(1.5$ to 5.1$)$ \\
\hline Anal sex with HIV-IUK partner always with a condom & 29.6 (25.0 to 34.2$)$ & $2.8(1.2$ to 4.5$)$ & $2.0(1.1$ to 3.7$)$ & & $2.2(0.7$ to 6.9$)$ \\
\hline Any condomless anal sex with HIV-/UK partner & $35.0(29.3$ to 40.7$)$ & $3.3(1.2$ to 5.5$)$ & $3.0(1.6$ to 5.3$)$ & & $1.7(0.6$ to 5.3$)$ \\
\hline \multicolumn{6}{|l|}{ Any recreational drug use in preceding 6 months 9} \\
\hline No & $21.1(19.2$ to 23.0$)$ & $0.9(0.4$ to 1.3$)$ & Reference & Reference & Reference \\
\hline Yes & 38.2 (33.7 to 42.8$)$ & 4.7 (2.8 to 6.7$)$ & $5.0(3.1$ to 5.9$)$ & 1.8 (1.1 to 3.2$)$ & $2.2(1.2$ to 3.9$)$ \\
\hline
\end{tabular}

UK=status unknown.

*Behaviours were time-updated at each completion of a sexual behaviour questionnaire.

tCumulative incidence proportions for testing and diagnosis were calculated using Kaplan-Meier methods.

¥Multivariable models adjusted for variables shown plus baseline age, region and time-updated viral load. Model A includes measures of sexual behaviours with HIV-negative,

HIV-positive and HIV status unknown partners grouped together. Model B distinguishes sexual behaviour according to partners' HIV status.

$\S$ The category 'sexually active, but no anal sex' was assigned to men who reported one or more partners to the question 'In the last 3 months how many male partners have you had

sex with?' but responded 'Never' to the statements 'I had anal sex with him' for any reported HIV-positive or HIV-negative/status unknown partner.

१Drug use was time -updated at each completion of a face-to-face interview. All drugs were combined to avoid multicollinearity.

MSM, men who have sex with men. 
among men who reported 5+ HIV-positive partners but not for multiple HIV-negative/unknown partners in the adjusted model. Recreational drug use doubled risk. Point estimates indicated elevated risk with anal sex (regardless of condom use); however, there was inadequate precision to indicate statistical significance.

\section{DISCUSSION}

Among gay and other MSM in an Ontario HIV-positive clinical cohort from 2010 to 2013, the 12-month cumulative incidence of a genital chlamydia/gonorrhoea diagnosis was substantial at $1.7 \%$ (95\% CI $1.1 \%$ to $2.2 \%$ ). Risk factors were having multiple HIV-positive partners and recreational drug use. Strengths of our analysis include a large sample, tests from a single laboratory and $98 \%$ completion rate of the sexual behaviour questionnaire. To our knowledge, our study is the first to compare longitudinal chlamydia/gonorrhoea diagnosis rates from multiple clinics among HIV-positive MSM by partners' HIV status.

There are limitations. Canadian and US guidelines recommend extragenital testing if engaging in oral or rectal intercourse $^{2}$ yet such tests were rarely done in our setting, meaning we could not identify rectal or pharyngeal infection risk factors. A 3-month time frame for sexual behaviours mitigated recall error but resulted in unmeasured behaviour between annual questionnaires, potentially explaining the non-zero diagnosis rate among men reporting no partners. Measurement of recreational drug use via face-to-face interviews may have introduced social desirability bias. Voluntary participation may have introduced selection bias. The OCS under-represents recent diagnoses; however, participants are generalisable to HIV-positive persons in Ontario according to sex, region, age at diagnosis and HIV exposure category. ${ }^{7}$

Recreational drug use was also a risk factor for chlamydia/ gonorrhoea diagnosis in cross-sectional prevalence studies executed in clinics in Madrid and the Netherlands, ${ }^{45}$ which was demonstrated for genital, rectal and pharyngeal infections. The Netherlands study reported multiple partners as a risk factor for chlamydia, gonorrhoea and syphilis combined; however, the association became null when syphilis and chlamydia/gonorrhoea were analysed separately. The Madrid study reported risk associated with serosorting, defined as having a stable HIV-positive partner, whereas in our setting having multiple HIV-positive partners was most important.

Only 5\% of men reported 5+ HIV-positive partners, but risk was highest in this group, with 12-month diagnosis rates of $8.3 \%$ (95\% CI $3.1 \%$ to $13.5 \%)$. After adjustment for HIV-positive partners, no additional risk was associated with having multiple HIV-negative/unknown partners. We propose three hypotheses. First, men may use condoms less frequently for 'poz' sex; however, we observed similar risks between condom users and non-users. Second, HIV-positive men may choose receptive anal sex with HIV-negative partners reducing their risk of genital infection; ${ }^{3}$ however, information on seropositioning was unavailable. Third, chlamydia/gonorrhoea may have achieved high prevalence in sexual networks among HIV-positive MSM seeking other positive men, and these networks may have low connectedness with HIV-negative MSM. That half of cases had a history of syphilis suggests presence of a core group where bacterial STIs are concentrated. In a study of German MSM, HIV-positive serosorters had threefold higher odds of bacterial STIs versus those with serodiscordant partners. ${ }^{8}$ Together with our finding of heightened risk for recreational drug use, we recommend further investigation of how 'scene' participation (eg, poz parties, bathhouses) influences STI risk. ${ }^{1}$ Further, as more HIV-negative men use pre-exposure prophylaxis for HIV prevention, sexual networks between HIV-negative and HIV-positive men may become more connected if serosorting becomes less common, ${ }^{9}$ resulting in more population-level transmission of bacterial STI.

Our findings have implications for chlamydia/gonorrhoea prevention, testing and treatment among HIV-positive MSM. Non-zero diagnosis rates among men reporting condomprotected anal sex suggests some acquisition through unmeasured risk practices such as oral sex; ${ }^{1}$ sexual education messages should emphasise the differences in bacterial STI transmission routes compared with HIV. Testing was below recommended guidelines; the proportion of untested men reporting high-risk behaviours was substantial. We strongly encourage rectal testing which could detect up to $80 \%$ more asymptomatic infections. ${ }^{5}$ There is a need to design and deliver interventions to promote safer sex and testing in ways that are engaging, non-stigmatising and acceptable for men at risk. Potential clinic-based interventions to optimise testing include algorithms targeting high-risk patients, clinical decision support systems, automated patient/ provider alerts and laboratory technologies to improve case detection. ${ }^{10}$

\section{Author affiliations}

${ }^{1}$ Centre for Research on Inner City Health, Li Ka Shing Knowledge Institute, St. Michael's Hospital, Toronto, Ontario, Canada

${ }^{2}$ Public Health Laboratories, Public Health Ontario, Toronto, Ontario, Canada ${ }^{3}$ Dalla Lana School of Public Health, University of Toronto, Toronto, Ontario, Canada ${ }^{4}$ Baycrest Health Science, Toronto, Ontario, Canada

${ }^{5}$ Ontario HIV Treatment Network, Toronto, Ontario, Canada

${ }^{6}$ Department of Medicine, University of Toronto, Toronto, Ontario, Canada

${ }^{7}$ Toronto General Research Institute, University Health Network, Toronto, Ontario, Canada

${ }^{8}$ Division of Infectious Diseases, St. Michael's Hospital, Toronto, Ontario, Canada

${ }^{9}$ Institute of Health Policy, Management and Evaluation, University of Toronto,

Toronto, Ontario, Canada

${ }^{10}$ Division of General Internal Medicine, St. Michael's Hospital, Toronto, Ontario, Canada

${ }^{11}$ Department of Microbiology, Mount Sinai Hospital/University Health Network, Toronto, Ontario, Canada

${ }^{12}$ AIDS Bureau, Ontario Ministry of Health and Long-Term Care, Toronto, Ontario, Canada

${ }^{13}$ Department of Psychiatry, University of Toronto, Toronto, Ontario, Canada

${ }^{14}$ Department of Family and Community Medicine, St. Michael's Hospital, Toronto, Ontario, Canada

\section{Handling editor Jackie A Cassell}

Acknowledgements The authors acknowledge the contribution of the late Dr Robert S Remis for guidance on earlier versions of this work. Further, we gratefully acknowledge all of the people living with HIV who volunteered to participate in the OHTN Cohort Study and the work and support of past and present members of the Governance Committee: Adrian Betts, Anita C. Benoit, Breklyn Bertozzi, Les Bowman, Lisungu Chieza, Tracey Conway, Patrick Cupido (Chair), Tony Di Pede, Brian Finch, Michael J. Hamilton, Brian Huskins, Rick Kennedy, Ken King, Nathan Lachowsky, Joanne Lindsay, Shari Margolese, Mark McCallum, John McTavish, Colleen Price, Lori Stoltz, Darien Taylor, Rosie Thein, and Drs AMB, Evan Collins, Curtis Cooper, Clemon George, Troy Grennan, Claire Kendall, and Greg Robinson. We thank all the interviewers, data collectors, research associates and coordinators, nurses and physicians who provide support for data collection and extraction. The authors wish to thank the OCS staff for data management, IT support, and study coordination (Madison Kopansky-Giles, Jason Globerman, Beth Rachlis, Robert Hudder, Gokul Kalaimani, Lucia Light, Veronika Moravan, Nahid Qureshi) and Public Health Ontario Laboratories for supporting record linkage with the HIV viral load, chlamydia and gonorrhoea test databases.

Collaborators The OHTN Cohort Study Team consists of Dr SBR (Principal Investigator), University of Toronto and OHTN; Dr Sergio Rueda, CAMH; Dr ANB, Dr AMB, Dr Kevin Gough and Dr DHST, St Michael's Hospital; Dr Jeffrey Cohen, Windsor Regional Hospital; Dr Curtis Cooper, Ottawa General Hospital; Dr Don Kilby, University of Ottawa Health Services; Dr Mona Loutfy and Dr Fred Crouzat, Maple Leaf Medical Clinic; Dr Anita Rachlis and Dr Nicole Mittmann, Sunnybrook Health Sciences Centre; Dr JR and Dr Irving Salit, Toronto General Hospital; Dr Edward Ralph, Dr Michael Silverman, St Joseph's Health Care; Dr Roger Sandre, 
Sudbury Regional Hospital; and Dr Gerald Evans and Dr Wendy Wobeser, Hotel Dieu Hospital.

Contributors RG conducted the analysis and wrote the manuscript. ANB was the principal investigator who conceived the project, obtained funding and contributed to the direction of the analysis and manuscript writing; she is the guarantor. ANB and SBR directed data collection at clinic sites. VGA and TM directed laboratory analysis at the PHOL and its interpretation. SG, VM and JR provided statistical expertise. AMB, RK, DHST and FM, contributed to the study protocol and guided interpretation. All authors provided critical input into the analysis, read an earlier version of the paper, provided substantive feedback and approved the final paper.

Funding This work was supported by Canadian Institutes of Health Research (CIHR) operating grant 111146, CIHR New Investigator awards to ANB and to DHST, an OHTN Chair and support from the Toronto and Western Hospital Foundation Skate the Dream Fund for JR and a Fondation Baxter and Alma Ricard Chair in Inner City Health at St Michael's Hospital for AMB. The OHTN Cohort Study is funded by the AIDS Bureau, Ontario Ministry of Health and Long-Term Care.

Disclaimer The opinions, results and conclusions are those of the authors and no endorsement by the Ontario HIV Treatment Network or Public Health Ontario is intended or should be inferred.

Competing interests None declared.

Ethics approval University of Toronto HIV Research Ethics Board (protocol reference 23954).

Provenance and peer review Not commissioned; externally peer reviewed.

Data sharing statement The OHTN may authorise the collection, use and disclosure of OCS data for the purpose of scholarly research if the proposal for the research project has been reviewed and approved and the research project principal investigator and all other members of the research project team have signed a researcher's agreement with the OHTN and adhere to all relevant OCS data and research policies.

Open Access This is an Open Access article distributed in accordance with the Creative Commons Attribution Non Commercial (CC BY-NC 4.0) license, which permits others to distribute, remix, adapt, build upon this work non-commercially, and license their derivative works on different terms, provided the original work is properly cited and the use is non-commercial. See: http://creativecommons.org/ licenses/by-nc/4.0/

\section{REFERENCES}

1 Public Health Agency of Canada. Section 5-Management and Treatment of Specific Infections \& Section 6-Specific Populations-Men Who Have Sex with Men (MSM)/Women Who Have Sex with Women (WSW). Canadian Guidelines on Sexually Transmitted Infections. Ottawa, ON: Public Health Agency of Canada, 2010.

2 Burchell AN, Grewal R, Allen VG, et al. Modest rise in chlamydia and gonorrhoea testing did not increase case detection in a clinical HIV cohort in Ontario, Canada. Sex Transm Infect 2014;90;608-14.

3 Cassels S, Katz DA. Seroadaptation among men who have sex with men: emerging research themes. Curr HIVIAIDS Rep 2013;10:305-13. 6

4 Ariza-Mejia M, Garcia-Garcia L, Puerta-Lopez T, et al. Risk factors of HIV co-infection and sexual behaviours in patients with Gonococcal infection in a STI's clinic in Madrid. J AIDS Clin Res 2013;4:240.

5 Heiligenberg M, Rijnders B, Schim van der Loeff MF, et al. High prevalence of sexually transmitted infections in HIV-infected men during routine outpatient visits in the Netherlands. Sex Transm Dis 2012;39:8-15.

6 Rourke SB, Gardner S, Burchell AN, et al. Cohort profile: the Ontario HIV Treatment Network Cohort Study (OCS). Int J Epidemiol 2013;42:402-11.

7 Raboud J, Su D, Burchell AN, et al. Representativeness of an HIV cohort of the sites from which it is recruiting: results from the Ontario HIV Treatment Network (OHTN) cohort study. BMC Med Res Methodol 2013;13:31.

8 Marcus U, Schmidt AJ, Hamouda O. HIV serosorting among HIV-positive men who have sex with men is associated with increased self-reported incidence of bacterial sexually transmissible infections. Sex Health 2011;8:184-93.

9 Grant RM, Smith DK. Integrating antiretroviral strategies for Human Immunodeficiency Virus prevention: post- and pre-exposure prophylaxis, and early treatment. Open Forum Infect Dis 2015;2:ofv126.

10 Fairley CK, Law M, Chen MY. Eradicating syphilis, hepatitis C and HIV in MSM through frequent testing strategies. Curr Opin Infect Dis 2014;27:56-61. 